SLAC-PUB-9055

November 2001

\title{
The Heisenberg Matrix Formulation of Quantum Field Theory
}

\author{
Stanley J. Brodsky \\ Stanford Linear Accelerator Center \\ Stanford University, Stanford, California 94309 \\ sjbth@slac.stanford.edu
}

\begin{abstract}
Presented at the
Symposium On 100 Years Werner Heisenberg: Works And Impact

Bamberg, Germany

26-30 September 2001
\end{abstract}

\footnotetext{
${ }^{*}$ Work supported by Department of Energy contract DE-AC03-76SF00515.
} 


\begin{abstract}
:
Heisenberg's matrix formulation of quantum mechanics can be generalized to relativistic systems by evolving in light-front time $\tau=t+z / c$. The spectrum and wavefunctions of bound states, such as hadrons in quantum chromodynamics, can be obtained from matrix diagonalization of the light-front Hamiltonian on a finite dimensional light-front Fock basis defined using periodic boundary conditions in $x^{-}$ and $x_{\perp}$. This method, discretized light-cone quantization (DLCQ), preserves the frame-independence of the front form even at finite resolution and particle number. Light-front quantization can also be used in the Hamiltonian form to construct an event generator for high energy physics reactions at the amplitude level. The lightfront partition function, summed over exponentially-weighted light-front energies, has simple boost properties which may be useful for studies in heavy ion collisions. I also review recent work which shows that the structure functions measured in deep inelastic lepton scattering are affected by final-state rescattering, thus modifying their connection to light-front probability distributions. In particular, the shadowing of nuclear structure functions is due to destructive interference effects from leadingtwist diffraction of the virtual photon, physics not included in the nuclear light-front wavefunctions.
\end{abstract}

\title{
1 Introduction
}

One of the challenges of relativistic quantum field theory is to compute the wavefunctions of bound states, such as the amplitudes which determine the quark and gluon substructure of hadrons in quantum chromodynamics. However, any extension of the Heisenberg-Schrödinger formulation of quantum mechanics $H|\psi\rangle=i \frac{\partial}{\partial t}|\psi\rangle=E|\psi\rangle$ to the relativistic domain has to confront seemingly intractable hurdles: (1) quantum fluctuations preclude finite particle-number wavefunction representations; (2) the charged particles arising from the quantum fluctuations of the vacuum contribute to the matrix element of currents - thus knowledge of the wavefunctions alone is insufficient to determine observables; and (3) the boost of an equal-time wavefunction from one Lorentz frame to another not only changes particle number, but is as complicated a dynamical problem as solving for the wavefunction itself.

In 1949, Dirac [1] made the remarkable observation that ordinary "instant" time $t$ is not the only possible evolution parameter. In fact, evolution in "light-front" time $\tau=t+z / c=x^{+}$has extraordinary advantages for relativistic systems, stemming from the fact that a subset of the Lorentz boost operators becomes purely kinematical. In fact, the Fock-state representation of bound states defined at equal light-front time, i.e., along the light-front, provides wavefunctions of fixed particle number which are independent of the eigenstate's four-momentum $P^{\mu}$. Furthermore, quantum fluctuations of the vacuum are absent if one uses light-front time to quantize the system, so that matrix elements such as the electromagnetic form factors only depend on the 


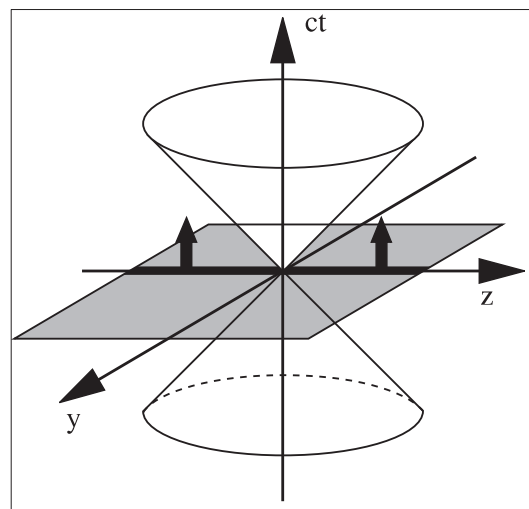

The instant form

$$
\begin{gathered}
\widetilde{\mathrm{x}}^{0}=\mathrm{ct} \\
\widetilde{\mathrm{x}}^{1}=\mathrm{x} \\
\widetilde{\mathrm{x}}^{2}=\mathrm{y} \\
\widetilde{\mathrm{x}}^{3}=\mathrm{z} \\
\widetilde{\mathrm{g}}_{\mu \nu}=\left(\begin{array}{rrrr}
1 & 0 & 0 & 0 \\
0 & -1 & 0 & 0 \\
0 & 0 & -1 & 0 \\
0 & 0 & 0 & -1
\end{array}\right)
\end{gathered}
$$

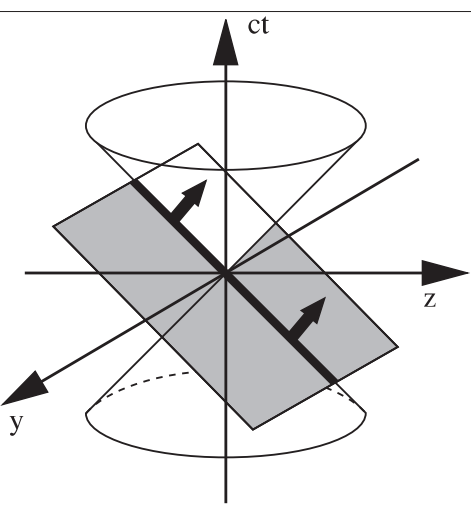

The front form

$$
\begin{gathered}
\tilde{\mathrm{x}}^{0}=\mathrm{ct}+\mathrm{z} \\
\tilde{\mathrm{x}}^{1}=\mathrm{x} \\
\tilde{\mathrm{x}}^{2}=\mathrm{y} \\
\tilde{\mathrm{x}}^{3}=\mathrm{ct}-\mathrm{z} \\
\widetilde{\mathrm{g}}_{\mu \nu}=\left(\begin{array}{rrrr}
0 & 0 & 0 & \frac{1}{2} \\
0 & -1 & 0 & 0 \\
0 & 0 & -1 & 0 \\
\frac{1}{2} & 0 & 0 & 0
\end{array}\right)
\end{gathered}
$$

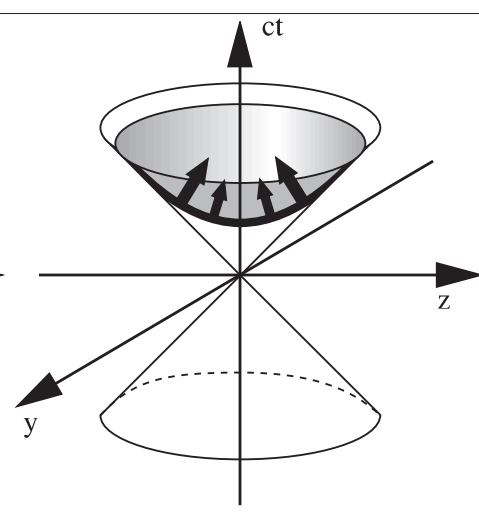

The point form

$$
\begin{aligned}
& \tilde{\mathrm{x}}^{0}=\tau, \quad \mathrm{ct}=\tau \cosh \omega \\
& \tilde{\mathrm{x}}^{1}=\omega, \quad \mathrm{x}=\tau \sinh \omega \sin \theta \cos \phi \\
& \tilde{\mathrm{x}}^{2}=\theta, \quad \mathrm{y}=\tau \sinh \omega \sin \theta \sin \phi \\
& \tilde{\mathrm{x}}^{3}=\phi, \quad \mathrm{x}=\tau \sinh \omega \cos \theta \\
& \tilde{\mathrm{g}}_{\mu \nu}=\left(\begin{array}{cccc}
1 & 0 & 0 & 0 \\
0 & -\tau^{2} & 0 & 0 \\
0 & 0 & -\tau^{2} \sinh ^{2} \omega & 0 \\
0 & 0 & 0 & -\tau^{2} \sinh ^{2} \omega \sin ^{2} \theta
\end{array}\right)
\end{aligned}
$$

Figure 1: Dirac's three forms of Hamiltonian dynamics. From Ref. [2].

currents of the constituents described by the light-front wavefunctions.

In Dirac's "Front Form", the generator of light-front time translations is $P^{-}=i \frac{\partial}{\partial \tau}$. Boundary conditions are set on the transverse plane labeled by $x_{\perp}$ and $x^{-}=z-c t$. See Fig. 1. Given the Lagrangian of a quantum field theory, $P^{-}$can be constructed as an operator on the Fock basis, the eigenstates of the free theory. (This method is also called "light-cone" quantization in the literature.) Since each particle in the Fock basis is on its mass shell, $k^{-} \equiv k^{0}-k^{3}=\frac{k_{\perp}^{2}+m^{2}}{k^{+}}$, and its energy $k^{0}=\frac{1}{2}\left(k^{+}+k^{-}\right)$ is positive, only particles with positive momenta $k^{+} \equiv k^{0}+k^{3} \geq 0$ can occur in the Fock basis. Since the total plus momentum $P^{+}=\sum_{n} k_{n}^{+}$is conserved, the light-front vacuum cannot have any particle content. The operator $H_{L C}=P^{+} P^{-}-P_{\perp}^{2}$, the "light-front Hamiltonian", is frame-independent.

The Heisenberg equation on the light-front is

$$
H_{L C}|\Psi\rangle=M^{2}|\Psi\rangle
$$

This can in principle be solved by diagonalizing the matrix $\left\langle n\left|H_{L C}\right| m\right\rangle$ on the free Fock basis: 2]

$$
\sum_{m}\left\langle n\left|H_{L C}\right| m\right\rangle\langle m \mid \psi\rangle=M^{2}\langle n \mid \Psi\rangle .
$$

The eigenvalues $\left\{M^{2}\right\}$ of $H_{L C}=H_{L C}^{0}+V_{L C}$ give the squared invariant masses of the bound and continuum spectrum of the theory. For example, the light-cone gauge interaction terms of QCD which are important for a meson are illustrated in Fig. 2. 


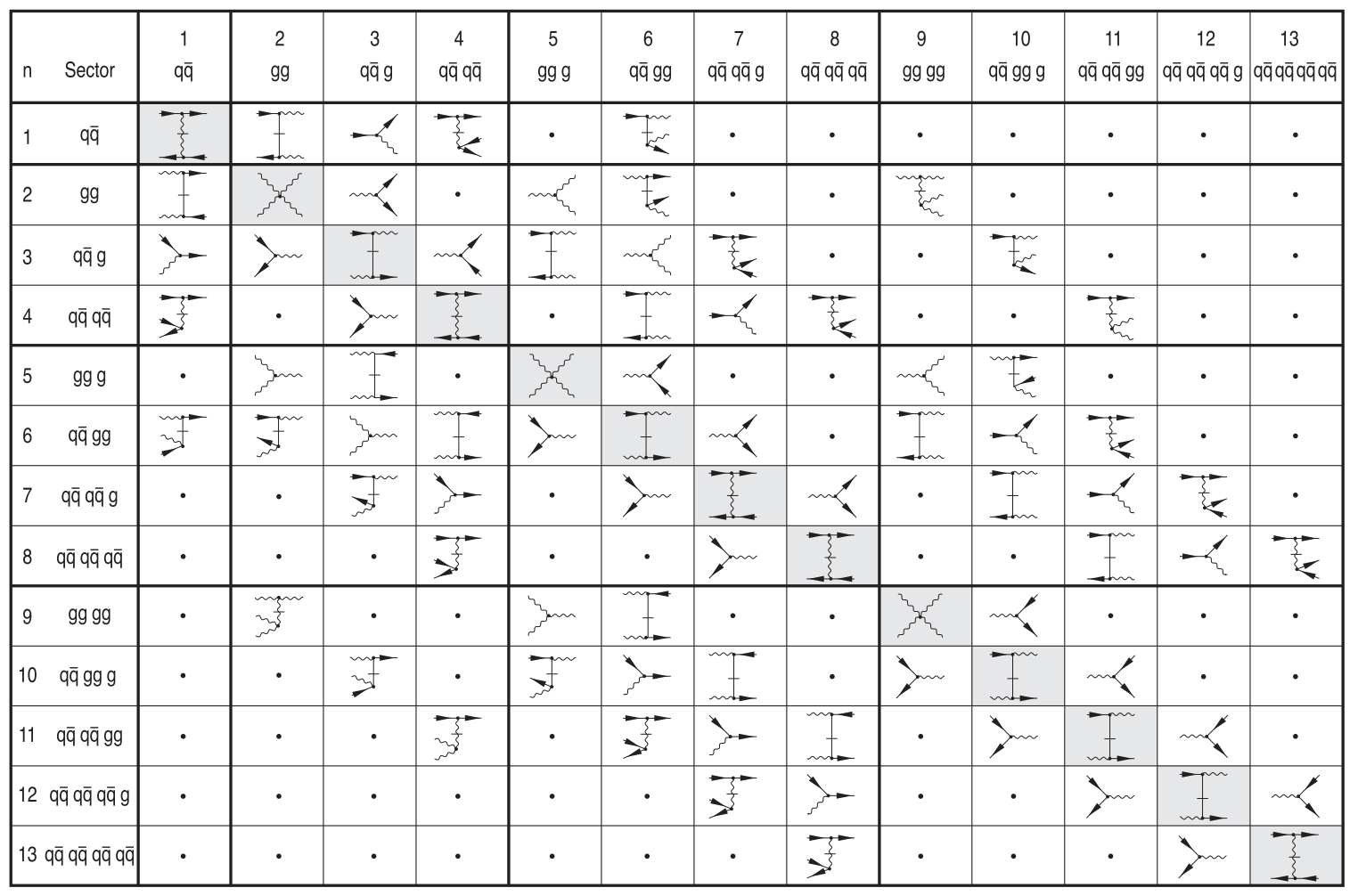

Figure 2: The front-form matrix of QCD interactions in light-cone gauge. Up to eight constituents in a meson are shown. From Ref. [2] and H. C. Pauli.

The projections $\{\langle n \mid \Psi\rangle\}$ of the eigensolution on the $n$-particle Fock states provide the light-front wavefunctions. Thus solving a quantum field theory is equivalent to solving a coupled many-body quantum mechanical problem:

$$
\left[M^{2}-\sum_{i=1}^{n} \frac{m_{\perp i}^{2}}{x_{i}}\right] \psi_{n}=\sum_{n^{\prime}} \int\left\langle n\left|V_{L C}\right| n^{\prime}\right\rangle \psi_{n^{\prime}}
$$

where the convolution and sum is understood over the Fock number, transverse momenta, plus momenta, and helicity of the intermediate states. Here $m_{\perp}^{2}=m^{2}+k_{\perp}^{2}$.

In QCD, the wavefunction of a hadron describes its composition in terms of the momenta and spin projections of quark and gluon constituents. For example, the eigensolution of a negatively-charged meson QCD, projected on its color-singlet $B=$ $0, Q=-1, J_{z}=0$ eigenstates $\{|n\rangle\}$ of the free Hamiltonian $H_{L C}^{Q C D}(g=0)$ at fixed $\tau=t-z / c$ has the expansion:

$$
\begin{aligned}
\left|\Psi_{M} ; P^{+}, \vec{P}_{\perp}, \lambda\right\rangle= & \sum_{n \geq 2, \lambda_{i}} \int \Pi_{i=1}^{n} \frac{d^{2} k_{\perp i} d x_{i}}{\sqrt{x_{i}} 16 \pi^{3}} 16 \pi^{3} \delta\left(1-\sum_{j}^{n} x_{j}\right) \delta^{(2)}\left(\sum_{\ell}^{n} \vec{k}_{\perp \ell}\right) \\
& \left|n ; x_{i} P^{+}, x_{i} \vec{P}_{\perp}+\vec{k}_{\perp i}, \lambda_{i}\right\rangle \psi_{n / M}\left(x_{i}, \vec{k}_{\perp i}, \lambda_{i}\right) .
\end{aligned}
$$


The set of light-front Fock state wavefunctions $\left\{\psi_{n / M}\right\}$ represent the ensemble of quark and gluon states possible when the meson is intercepted at the light-front. The light-front momentum fractions $x_{i}=k_{i}^{+} / P_{\pi}^{+}=\left(k^{0}+k_{i}^{z}\right) /\left(P^{0}+P^{z}\right)$ with $\sum_{i=1}^{n} x_{i}=1$ and $\vec{k}_{\perp i}$ with $\sum_{i=1}^{n} \vec{k}_{\perp i}=\overrightarrow{0}_{\perp}$ represent the relative momentum coordinates of the QCD constituents and are independent of the total momentum of the state. The actual physical transverse momenta are $\vec{p}_{\perp i}=x_{i} \vec{P}_{\perp}+\vec{k}_{\perp i}$. The $\lambda_{i}$ label the light-front spin $S^{z}$ projections of the quarks and gluons along the quantization $z$ direction. The spinors of the light-front formalism automatically incorporate the Melosh-Wigner rotation. The physical gluon polarization vectors $\epsilon^{\mu}(k, \lambda= \pm 1)$ are specified in light-cone gauge by the conditions $k \cdot \epsilon=0, \eta \cdot \epsilon=\epsilon^{+}=0$. The parton degrees of freedom are thus all physical; there are no ghost or negative metric states. A detailed derivation of light-front quantization of non-Abelian gauge theory in light-cone gauge is given in Ref. [3]. Explicit examples of light-front wavefunctions in QED are given in Ref. [4.

Angular momentum has simplifying features in the light-front formalism since the projection $J_{z}$ is kinematical and conserved. Each light-front Fock wavefunction satisfies the angular momentum sum rule: $J^{z}=\sum_{i=1}^{n} S_{i}^{z}+\sum_{j=1}^{n-1} l_{j}^{z}$. The sum over $S_{i}^{z}$ represents the contribution of the intrinsic spins of the $n$ Fock state constituents. The sum over orbital angular momenta

$$
l_{j}^{z}=-\mathrm{i}\left(k_{j}^{1} \frac{\partial}{\partial k_{j}^{2}}-k_{j}^{2} \frac{\partial}{\partial k_{j}^{1}}\right)
$$

derives from the $n-1$ relative momenta. This excludes the contribution to the orbital angular momentum due to the motion of the center of mass, which is not an intrinsic property of the hadron [4]. The numerator structure of the light-front wavefunctions is in large part determined by the angular momentum constraints.

The most important feature of light-front Fock wavefunctions $\psi_{n / p}\left(x_{i}, \vec{k}_{\perp i}, \lambda_{i}\right)$ is the fact they are Lorentz invariant functions of the relative coordinates, independent of the bound state's physical momentum $P^{+}=P^{0}+P^{z}$, and $P_{\perp}$ [5]. The lightfront wavefunctions represent the ensembles of states possible when the hadron is intercepted by a light-front at fixed $\tau=t+z / c$. The light-front representation thus provide a frame-independent, quantum-mechanical representation of a hadron at the amplitude level, capable of encoding its multi-quark, hidden-color and gluon momentum, helicity, and flavor correlations in the form of universal process-independent hadron wavefunctions.

If one imposes periodic boundary conditions in $x^{-}=t+z / c$, then the plus momenta become discrete: $k_{i}^{+}=\frac{2 \pi}{L} n_{i}, P^{+}=\frac{2 \pi}{L} K$, where $\sum_{i} n_{i}=K$ [6, 7]. For a given "harmonic resolution" $K$, there are only a finite number of ways positive integers $n_{i}$ can sum to a positive integer $K$. Thus at a given $K$, the dimension of the resulting light-front Fock state representation of the bound state is rendered finite without violating Lorentz invariance. The eigensolutions of a quantum field theory, both the bound states and continuum solutions, can then be found by nu- 
merically diagonalizing a frame-independent light-front Hamiltonian $H_{L C}$ on a finite and discrete momentum-space Fock basis. Solving a quantum field theory at fixed light-front time $\tau$ thus can be formulated as a relativistic extension of Heisenberg's matrix mechanics. The continuum limit is reached for $K \rightarrow \infty$. This formulation of the non-perturbative light-front quantization problem is called "discretized light-front quantization" (DLCQ) [7]. Lattice gauge theory has also been used to calculate the pion light-front wavefunction [8].

The DLCQ method has been used extensively for solving one-space and one-time theories [2], including applications to supersymmetric quantum field theories [9] and specific tests of the Maldacena conjecture [10]. There has been progress in systematically developing the computation and renormalization methods needed to make DLCQ viable for QCD in physical spacetime. For example, John Hiller, Gary McCartor, and I [11] have shown how DLCQ can be used to solve $3+1$ theories despite the large numbers of degrees of freedom needed to enumerate the Fock basis. A key feature of our work is the introduction of Pauli Villars fields to regulate the UV divergences and perform renormalization while preserving the frame-independence of the theory. A recent application of DLCQ to a $3+1$ quantum field theory with Yukawa interactions is given in Ref. [11]. Representative plots of the one-boson one-fermion light-front Fock wavefunction of the lowest mass fermion solution of the Yukawa $(3+1)$ theory showing spin correlations and the presence of non-zero orbital angular momentum are shown in Fig. 3.

There has also been important progress using the transverse lattice, essentially a combination of DLCQ in $1+1$ dimensions together with a lattice in the transverse dimensions [12, 13, 14]. One can also define a truncated theory by eliminating the higher Fock states in favor of an effective potential [15]. Spontaneous symmetry breaking and other nonperturbative effects associated with the instant-time vacuum are hidden in dynamical or constrained zero modes on the light-front. An introduction is given in Refs. [16, 17].

\section{General Features of Light-front Wavefunctions}

The maximum of a light-front wavefunction occurs when the invariant mass of the partons is minimal; i.e., when all particles have equal rapidity and are all at rest in the rest frame. In fact, Dae Sung Hwang and I [18] have noted that one can rewrite the wavefunction in the form:

$$
\psi_{n}=\frac{\Gamma_{n}}{M^{2}\left[\sum_{i=1}^{n} \frac{\left(x_{i}-\widehat{x}_{i}\right)^{2}}{x_{i}}+\delta^{2}\right]}
$$

where $x_{i}=\widehat{x}_{i} \equiv m_{\perp i} / \sum_{i=1}^{n} m_{\perp i}$ is the condition for minimal rapidity differences of the constituents. The key parameter is $M^{2}-\sum_{i=1}^{n} m_{\perp i}^{2} / \widehat{x}_{i} \equiv-M^{2} \delta^{2}$. One can interpret $\delta^{2} \simeq 2 \epsilon / M$ where $\epsilon=\sum_{i=1}^{n} m_{\perp i}-M$ is the effective binding energy. This 

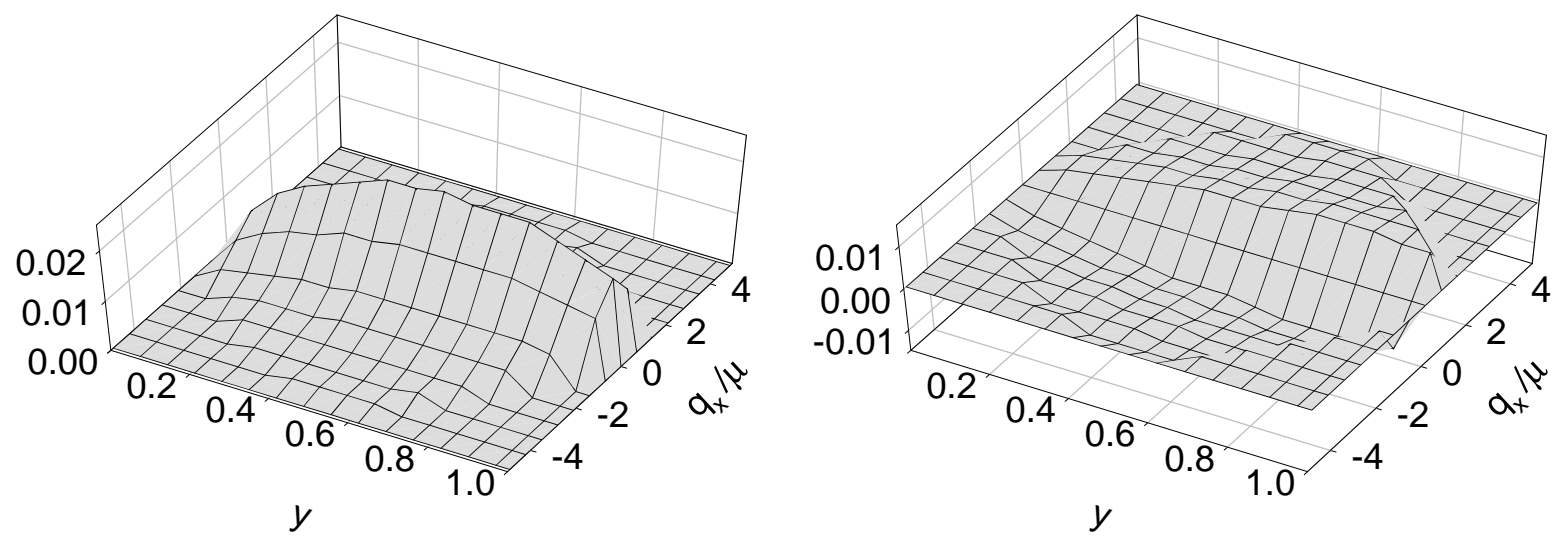

Figure 3: DLCQ results for the one-boson one-fermion wavefunction in a fermion system with parallel and antiparallel fermion helicity, as a function of longitudinal momentum fraction $y$ and one transverse momentum component $q_{x}$ in the $q_{y}=0$ plane. The parameter values for the DLCQ resolution are $K=29, N_{\perp}=7$. Further details are given in Ref. [11].

form shows that the wavefunction is a quadratic form around its maximum, and that the width of the distribution in $\left(x_{i}-\widehat{x}_{i}\right)^{2}$ (where the wavefunction falls to half of its maximum) is controlled by $x_{i} \delta^{2}$ and the transverse momenta $k_{\perp_{i}}$. Note also that the heaviest particles tend to have the largest $\widehat{x}_{i}$, and thus the largest momentum fraction of the particles in the Fock state, a feature familiar from the intrinsic charm model. For example, the $b$ quark has the largest momentum fraction at small $k_{\perp}$ in the $B$ meson's valence light-front wavefunction, but the distribution spreads out to an asymptotically symmetric distribution around $x_{b} \sim 1 / 2$ when $k_{\perp} \gg m_{b}^{2}$.

The fall-off the light-front wavefunctions at large $k_{\perp}$ and $x \rightarrow 1$ is dictated by QCD perturbation theory since the state is far-off the light-front energy shell. This leads to counting rule behavior for the quark and gluon distributions at $x \rightarrow 1$. Notice that $x \rightarrow 1$ corresponds to $k^{z} \rightarrow-\infty$ for any constituent with nonzero mass or transverse momentum.

The above discussion suggests that an approximate form for the hadron light-front wavefunctions could be constructed through variational principles and by minimizing the expectation value of $H_{L C}^{Q C D}$.

\section{A Light-front Event Amplitude Generator}

The light-front formalism can be used as an "event amplitude generator" for high energy physics reactions where each particle's final state is completely labeled in momentum, helicity, and phase. The application of the light-front time evolution operator $P^{-}$to an initial state systematically generates the tree and virtual loop graphs 
of the $T$-matrix in light-front time-ordered perturbation theory in light-front gauge. The loop integrals only involve integrations over the momenta of physical quanta and physical phase space $\prod d^{2} k_{\perp i} d k_{i}^{+}$. Renormalized amplitudes can be explicitly constructed by subtracting from the divergent loops amplitudes with nearly identical integrands corresponding to the contribution of the relevant mass and coupling counter terms (the "alternating denominator method") [19]. The natural renormalization scheme to use for defining the coupling in the event amplitude generator is a physical effective charge such as the pinch scheme [20]. The argument of the coupling is then unambiguous [21]. The DLCQ boundary conditions can be used to discretize the phase space and limit the number of contributing intermediate states without violating Lorentz invariance. Since one avoids dimensional regularization and nonphysical ghost degrees of freedom, this method of generating events at the amplitude level could provide a simple but powerful tool for simulating events both in QCD and the Standard Model.

\section{The Light-front Partition Function}

In the usual treatment of classical thermodynamics, one considers an ensemble of particles $n=1,2, \ldots N$ which have energies $\left\{E_{n}\right\}$ at a given "instant" time $t$. The partition function is defined as $Z=\sum_{n} \exp -\frac{E_{n}}{k T}$. Similarly, in quantum mechanics, one defines a quantum-statistical partition function as $Z=\operatorname{tr} \exp -\beta H$ which sums over the exponentiated-weighted energy eigenvalues of the system.

In the case of relativistic systems, it is natural to characterize the system at a given light-front time $\tau=t+z / c$; i.e., one determines the state of each particle in the ensemble as its encounters the light-front. Thus we can define a light-front partition function

$$
Z_{L C}=\sum_{n} \exp -\frac{p_{n}^{-}}{k T_{L C}}
$$

by summing over the particles' light-front energies $p^{-}=p^{0}-p^{z}=\frac{p_{\perp}^{2}+m^{2}}{p^{+}}$. The total momentum is $P^{+}=\sum p_{n}^{+}, \vec{P}_{\perp}=\sum_{n} \vec{p}_{\perp n}$, and the total mass is defined from $P^{+} P^{-}-P_{\perp}^{2}=M^{2}$. The product $\frac{M}{P^{-}} T_{L C}$ is boost invariant. In the center of mass frame where $\vec{P}=0$ and thus $P^{+}=P^{-}=M$. It is also possible to consistently impose boundary conditions at fixed $x^{-}=z-c t$ and $x_{\perp}$, as in DLCQ. The momenta $p_{n}^{+}, \vec{p}_{\perp n}$ then become discrete. The corresponding light-front quantum-statistical partition function is $Z=\operatorname{tr} \exp -\beta_{L C} H_{L C}$ where $H_{L C}=i \frac{\partial}{\partial \tau}$ is the light-front Hamiltonian.

For non-relativistic systems the light-front partition function reduces to the standard definition. However, the light-front partition function should be advantageous for analyzing relativistic systems such as heavy ion collisions, since, like true rapidity, $y=\ln \frac{p^{+}}{P^{+}}$, light-front variables have simple behavior under Lorentz boosts. The light-front formalism also takes into account the point that a phase transition does 
not occur simultaneously in $t$, but propagates through the system with a finite wave velocity.

\section{Light-front Wavefunctions and QCD Phenomenol- ogy}

There have been extensive applications of light-front wavefunctions to QCD phenomenology [22]; for example, form factors [23] and the handbag contribution to deeply virtual Compton scattering $\gamma^{*} p \rightarrow \gamma p$ can be expressed as overlaps of the light-front wavefunctions [24, 25]; quark and gluon distributions are light-front wavefunction probabilities. The distributions measured in the diffractive dissociation of hadrons are computed from transverse derivatives of the light-front wavefunctions. Progress in measuring the basic parameters of electroweak interactions and $C P$ violation will require a quantitative understanding of the dynamics and phase structure of $B$ decays at the amplitude level. The light-front Fock representation is specially advantageous in the study of exclusive $B$ decays. For example, Dae Sung Hwang [26] and I have derived an exact frame-independent representation of decay matrix elements such as $B \rightarrow D \ell \bar{\nu}$ from the overlap of $n^{\prime}=n$ parton-number conserving wavefunctions and the overlap of wavefunctions with $n^{\prime}=n-2$ from the annihilation of a quark-antiquark pair in the initial wavefunction.

One can also express the matrix elements of the energy momentum tensor as overlap integrals of the light-front wavefunctions [4]. An important consistency check of any relativistic formalism is to verify the vanishing of the anomalous gravitomagnetic moment $B(0)$, the spin-flip matrix element of the graviton coupling and analog of the anomalous magnetic moment $F_{2}(0)$. For example, at one-loop order in QED, $B_{f}(0)=\frac{\alpha}{3 \pi}$ for the electron when the graviton interacts with the fermion

line, and $B_{\gamma}(0)=-\frac{\alpha}{3 \pi}$ when the graviton interacts with the exchanged photon. The vanishing of $B(0)$ can be shown to be exact for bound or elementary systems in the light-front formalism [4], in agreement with the equivalence principle [27, 28, 29].

\section{Structure Functions are Not Parton Distribu- tions}

The quark and gluon distributions of hadrons can be defined from the probability measures of the light-front wavefunctions. For example, the quark distribution in a hadron $H$ is

$$
P_{\mathrm{q} / H}\left(x_{B j}, Q^{2}\right)=\sum_{n} \int^{k_{i \perp}^{2}<Q^{2}}\left[\prod_{i} d x_{i} d^{2} k_{\perp i}\right]\left|\psi_{n / H}\left(x_{i}, k_{\perp i}\right)\right|^{2} \sum_{j=q} \delta\left(x_{B j}-x_{j}\right) .
$$


It has been conventional to identify the leading-twist structure functions $F_{i}\left(x, Q^{2}\right)$ measured in deep inelastic lepton scattering with the light-front probability distributions. For example, in the parton model, $F_{2}\left(x, Q^{2}\right)=\sum_{q} e_{q}^{2} P_{\mathrm{q} / H}\left(x, Q^{2}\right)$. However, Paul Hoyer, Nils Marchal, Stephane Peigne, Francesco Sannino, and I [30] have recently shown that the leading-twist contribution to deep inelastic scattering is affected by diffractive rescattering of a quark in the target, a coherent effect which is not included in the light-front wavefunctions, even in light-cone gauge. The gluon propagator in light-cone gauge $A^{+}=0$ is singular:

$$
d_{L C}^{\mu \nu}(k)=\frac{i}{k^{2}+i \varepsilon}\left[-g^{\mu \nu}+\frac{n^{\mu} k^{\nu}+k^{\mu} n^{\nu}}{n \cdot k}\right]
$$

has a pole at $k^{+} \equiv n \cdot k=0$, which has to be defined by an analytic prescription such as the Mandelstam-Liebbrandt prescription [31]. In final-state scattering involving on-shell intermediate states, the exchanged momentum $k^{+}$is of $O(1 / \nu)$ in the target rest frame, which enhances the second term of the light-cone gauge propagator. This enhancement allows rescattering to contribute at leading twist even in LC gauge.

Thus diffractive contributions to the deep inelastic scattering $\gamma^{*} p \rightarrow X p^{\prime}$ cross sections, which leave the target intact, contribute at leading twist to deep inelastic scattering. Diffractive events resolve the quark-gluon structure of the virtual photon, not the quark-gluon structure of the target, and thus they give contributions to structure functions which are not target parton probabilities. Our analysis of deep inelastic scattering $\gamma^{*}(q) p \rightarrow X$, when interpreted in frames with $q^{+}>0$, also supports the color dipole description of deep inelastic lepton scattering at small $x_{b j}$. For example, in the case of the aligned-jet configurations, one can understand $\sigma_{T}\left(\gamma^{*} p\right)$ at high energies as due to the coherent color gauge interactions of the incoming quarkpair state of the photon interacting, first coherently and finally incoherently, in the target.

The distinction between structure functions and target parton probabilities is also implied by the Glauber-Gribov picture of nuclear shadowing [32, 33, 34, 355. In this framework, shadowing arises from interference between complex rescattering amplitudes involving on-shell intermediate states. In contrast, the wave function of a stable target is strictly real since it does not have on energy-shell configurations. Thus nuclear shadowing is not a property of the light-front wavefunctions of a nuclear target; rather, it involves the total dynamics of the $\gamma^{*}$ - nucleus collision. A strictly probabilistic interpretation of the deep inelastic cross section cross section is thus precluded.

\section{Acknowledgment}

I thank Professor Harald Fritzsch and the Von Humboldt Foundation for inviting me to the Heisenberg Symposium. Much of the work reported here was done in collaboration with others, especially, Markus Diehl, John Hiller, Paul Hoyer, Dae Sung Hwang, 
Nils Marchal, Bo-Qiang Ma, Gary McCartor, Hans Christian Pauli, Stephane Peigne, Francesco Sannino, Ivan Schmidt, and Prem Srivastava. This work was supported by the Department of Energy under contract number DE-AC03-76SF00515.

\section{References}

[1] P. A. Dirac, Rev. Mod. Phys. 21, 392 (1949).

[2] For a review and references, see S. J. Brodsky, H. C. Pauli and S. S. Pinsky, Phys. Rept. 301, 299 (1998) arXiv:hep-ph/9705477.

[3] P. P. Srivastava and S. J. Brodsky, Phys. Rev. D 64, 045006 (2001) arXiv:hepph/0011372].

[4] S. J. Brodsky, D. S. Hwang, B. Ma and I. Schmidt, Nucl. Phys. B 593, 311 (2001) hep-th/0003082.

[5] G. P. Lepage and S. J. Brodsky, Phys. Rev. D 22, 2157 (1980).

[6] T. Maskawa and K. Yamawaki, Method Of Quantization," Prog. Theor. Phys. 56, 270 (1976).

[7] H. C. Pauli and S. J. Brodsky, Phys. Rev. D 32, 1993 (1985).

[8] A. Abada, P. Boucaud, G. Herdoiza, J. P. Leroy, J. Micheli, O. Pene and J. Rodriguez-Quintero, Phys. Rev. D 64, 074511 (2001) arXiv:hep-ph/0105221.

[9] Y. Matsumura, N. Sakai and T. Sakai, Phys. Rev. D 52, 2446 (1995) arXiv:hepth/9504150].

[10] J. R. Hiller, S. Pinsky and U. Trittmann, supersymmetric Yang-Mills theory," Phys. Rev. D 64, 105027 (2001) arXiv:hep-th/0106193.

[11] S. J. Brodsky, J. R. Hiller and G. McCartor, hep-ph/0107038.

[12] W. A. Bardeen, R. B. Pearson and E. Rabinovici, Phys. Rev. D 21, 1037 (1980).

[13] S. Dalley, Phys. Rev. D 64, 036006 (2001) arXiv:hep-ph/0101318.

[14] M. Burkardt and S. K. Seal, arXiv:hep-ph/0105109.

[15] H. C. Pauli, arXiv:hep-ph/0111040.

[16] G. McCartor, in Proc. of New Nonperturbative Methods and Quantization of the Light Cone, Les Houches, France, 24 Feb - 7 Mar 1997.

[17] K. Yamawaki, arXiv:hep-th/9802037. 
[18] S. J. Brodsky and D.-S. Hwang, in preparation.

[19] S. J. Brodsky, R. Roskies and R. Suaya, Momentum Frame," Phys. Rev. D 8, 4574 (1973).

[20] J. M. Cornwall and J. Papavassiliou, Phys. Rev. D 40, 3474 (1989).

[21] S. J. Brodsky and H. J. Lu, Phys. Rev. D 51, 3652 (1995) arXiv:hepph/9405218.

[22] S. J. Brodsky, arXiv:hep-ph/0102051.

[23] S. J. Brodsky and S. D. Drell, Phys. Rev. D 22, 2236 (1980).

[24] S. J. Brodsky, M. Diehl and D. S. Hwang, Nucl. Phys. B 596, 99 (2001) arXiv:hep-ph/0009254.

[25] M. Diehl, T. Feldmann, R. Jakob and P. Kroll, Nucl. Phys. B 596, 33 (2001) [Erratum-ibid. B 605, 647 (2001)] [arXiv:hep-ph/0009255].

[26] S. J. Brodsky and D. S. Hwang, Nucl. Phys. B 543, 239 (1999) hep-ph/9806358.

[27] L. Okun and I. Yu. Kobsarev, ZhETF, 43 (1962) 1904 (English translation: JETP 16 (1963) 1343); L. Okun, in proceedings of the 4th International Conference on Elementary Particles, Heidelberg, Germany (1967), edited by H. Filthuth, North-Holland (1968).

[28] I. Yu. Kobsarev and V. I. Zakharov, Ann. Phys. 60 (1970) 448.

[29] O. V. Teryaev, hep-ph/9904376 (1999).

[30] S. J. Brodsky, P. Hoyer, N. Marchal, S. Peigne and F. Sannino, hep-ph/0104291.

[31] G. Leibbrandt, Rev. Mod. Phys. 59, 1067 (1987).

[32] V. N. Gribov, Sov. Phys. JETP 29, 483 (1969) [Zh. Eksp. Teor. Fiz. 56, 892 (1969)].

[33] S. J. Brodsky and J. Pumplin, Phys. Rev. 182, 1794 (1969).

[34] S. J. Brodsky and H. J. Lu, Phys. Rev. Lett. 64, 1342 (1990).

[35] G. Piller and W. Weise, Phys. Rept. 330, 1 (2000) [hep-ph/9908230. 